\title{
Utilização e cobertura do Sistema de Vigilância Alimentar e Nutricional no Estado do Rio Grande do Sul, Brasil
}

\author{
Utilization and coverage of a Food and Nutritional Surveillance \\ System in Rio Grande do Sul state, Brazil
}

Natália Miranda Jung ${ }^{1}$

Fernanda de Souza Bairros ${ }^{2}$

Marilda Borges Neutzling ${ }^{2}$

\footnotetext{
${ }^{1}$ Grupo Hospitalar Conceição. Av. Francisco Trein 596, Bairro Cristo Redentor. 91.350-200 Porto Alegre RS Brasil. nataliajung@ghc.com.br ${ }^{2}$ Departamento de Medicina Social, Faculdade de Medicina, Universidade Federal do Rio Grande do Sul.
}

\begin{abstract}
This article seeks to describe the utilization and coverage percentage of the Nutritional and Food Surveillance System (SISVAN-Web) in the Regional Health Offices of Rio Grande do Sul in 2010 and to assess its correlation with socio-economic, demographic and health system organization variables at the time. It is an ecological study that used secondary data from the SISVAN-Web, the Department of Primary Health Care, the IT Department of the Unified Health System and the Brazilian Institute of Geography and Statistics. The evaluation of utilization and coverage data was restricted to nutritional status. The percentage of utilization of SISVAN-Web refers to the number of cities that fed the system. Total coverage was defined as the percentage of individuals in all stages of the life cycle monitored by SISVAN-Web. It was found that 324 cities fed the application, corresponding to a utilization percentage of $65.3 \%$. Greater system coverage was observed in all Regional Health Coordination (RHC) Units for ages 0 to 5 years and 5-10 years. There was a significant association between the percentage of utilization of SISVAN-Web and Family Health Strategy coverage in each RHC Unit. The results of this study indicated low percentages of utilization and coverage of SISVANWeb in Rio Grande do Sul.

Key words Nutritional and food surveillance, Coverage, Nutritional assessment
\end{abstract}

Resumo O objetivo deste artigo é descrever o percentual de utilização e a cobertura do Sistema de Vigilância Alimentar e Nutricional - Web (SISVAN-Web) nas Coordenadorias Regionais de Saúde do Rio Grande do Sul em 2010 e verificar sua correlação com variáveis socioeconômicas, demográficas e de organização do sistema de saúde. Estudo ecológico que utilizou dados secundários do SISVAN-Web, Departamento de Atenção Básica do Ministério da Saúde, Departamento de Informática do Sistema Único de Saúde e Instituto Brasileiro de Geografia e Estatística. A avaliação de utilização e cobertura restringiu-se aos dados de estado nutricional. Percentual de utilização refere-se ao número de municípios que alimentaram o sistema. Cobertura total foi definida como o percentual de indivíduos acompanhados pelo SISVAN-Web. Verificou-se que 324 municipios do $R S$ alimentaram o aplicativo, correspondendo a um percentual de utilização de 65,3\%. Observouse em todas as CRS uma maior cobertura do sistema nas idades de 0 a 5 anos e 5 a 10 anos. Verificou-se relação significativa entre o percentual de utilização do SISVAN-Web e a cobertura da Estratégia de Saúde da Família (ESF) em cada CRS. Os resultados deste estudo indicaram baixos percentuais de utilização e cobertura do SISVANWeb no Rio Grande do Sul em 2010.

Palavras-chave Vigilância alimentar e nutricional, Cobertura, Avaliação nutricional 


\section{Introdução}

A população brasileira, nas últimas décadas, experimentou transformações no padrão de saúde e consumo alimentar ${ }^{1}$. No contexto da transição alimentar e nutricional, dois extremos compartilham o mesmo cenário. De um lado, observase o aumento vertiginoso do excesso de peso e, de outro, a manutenção de elevadas frequências de carências nutricionais específicas ${ }^{2-5}$. A Política Nacional de Alimentação e Nutrição (PNAN) brasileira constitui-se em um compromisso do Ministério da Saúde (MS) frente à necessidade de enfrentamento de tal paradoxo nutricional ${ }^{1,6}$.

Apesar do consenso acerca da importância de avaliações sistemáticas das políticas públicas ${ }^{7}$, as avaliações em saúde são raras e sua institucionalização constitui-se em um grande desafio ${ }^{8,9}$. Programas podem ser ineficazes simplesmente porque sua cobertura é pouco expressiva para impactar sobre a situação-problema ${ }^{10}$. Frente a isso, a documentação de indicadores de processo (oferta, utilização e cobertura) torna-se importante para o processo gerencial, entendido como prática reflexiva de acompanhamento das intervenções, de modo a prover a administração de informações sintéticas que subsidiem o processo de tomada de decisões ${ }^{9,11}$.

A Vigilância Alimentar e Nutricional (VAN), terceira diretriz da PNAN, constitui-se em elemento central no monitoramento da situação alimentar e nutricional da população ${ }^{12}$. Entende-se que a VAN deve contemplar diferentes estratégias de vigilância epidemiológica. Os inquéritos populacionais, as chamadas nutricionais e a produção científica podem ser citados como exemplos ${ }^{1,6,13}$. Segundo Leal e Bittencourt ${ }^{2}$, o alto custo, a demora na publicação dos resultados, a falta de representatividade em nível local e a intermitência das informações geradas por essas estratégias mostrou-se insuficiente para subsidiar as políticas de saúde e nutrição. Assim, na década de 1990, foi instituído o Sistema de Vigilância Alimentar e Nutricional (SISVAN).

Lançado em dezembro de 2007, o SISVANWeb, atual sistema informatizado de VAN, apresenta como vantagens em relação ao sistema anterior, conhecido como "módulo municipal", a apresentação de uma interface mais fácil para o usuário do sistema ${ }^{6}$. Operado a partir da Atenção Primária à Saúde (APS), possui como objetivo monitorar o estado nutricional e o padrão alimentar dos indivíduos atendidos pelo SUS ${ }^{1}$. Para que seja oportuno em suas conclusões e recomendações, assim como todo sistema de vigi- lância epidemiológica, o SISVAN deve possuir cobertura suficiente e propósitos claramente definidos $^{14}$.

Ainda são poucos os estudos publicados que avaliam o desempenho do SISVAN, especificamente no que diz respeito à utilização e cobertura desse aplicativo nas três esferas de gestão do SUS. O estado nutricional da população é um indicador positivo de saúde, ao contrário da maioria dos indicadores clássicos de morbidade e mortalidade, que medem a ausência de saúde ${ }^{2}$. Dessa forma, conhecer a utilização e a cobertura do sistema é de fundamental importância para avaliar a representatividade dos indicadores de alimentação e nutrição produzidos pelo SISVAN. O presente estudo propõe-se a descrever a utilização e a cobertura do SISVAN-Web nas dezenove Coordenadorias Regionais de Saúde (CRS) do Estado do Rio Grande do Sul no ano de 2010 e verificar sua correlação com variáveis socioeconômicas, demográficas e de organização do sistema de saúde no mesmo local e período.

\section{Metodologia}

O Rio Grande do Sul (RS), estado brasileiro localizado na região sul do país, é formado por 496 municípios. A população estimada pelo Censo 2010 foi de 10.693.929 habitantes, sendo considerado o quinto estado mais populoso do Brasil. A área mais densamente povoada do RS é a da capital Porto Alegre e Região Metropolitana, onde se concentra a quarta maior aglomeração urbana do país. Na área da saúde, o RS está dividido, político-administrativamente, em dezenove Coordenadorias Regionais de Saúde (CRS), que contêm equipe técnica e administrativa responsável pelo planejamento, acompanhamento e gerenciamento das ações e serviços de saúde locais e regionais, segundo os princípios do SUS ${ }^{15}$.

O presente estudo, de delineamento ecológico, utilizou dados secundários do Sistema de Vigilância Alimentar e Nutricional - Web (SISVANWeb $)^{16}$, Departamento de Atenção Básica do Ministério da Saúde (DAB/MS) ${ }^{17}$, Departamento de Informática do SUS (DATASUS) ${ }^{18}$ e Instituto Brasileiro de Geografia e Estatística (IBGE) ${ }^{19}$ no ano de 2010. Considerou-se como unidade de análise o universo das dezenove CRS $(\mathrm{n}=19)$ do RS.

Embora o Módulo Gerenciador de Relatórios do SISVAN-Web disponibilize dados de estado nutricional (EN) e consumo alimentar, optou-se por analisar apenas aqueles referentes ao EN da população acompanhada pelo sistema. A 
possibilidade de registro de marcadores do consumo alimentar ainda é bastante incipiente uma vez que teve início em 2007 quando ocorreu a transição do sistema anterior, conhecido como "módulo municipal", para o atual. Por ocorrer desde os primórdios da implantação do SISVAN, a coleta e a digitação de dados antropométricos têm sido preponderantes. Assim, entendeu-se que sua utilização e cobertura poderiam estar mais estabelecidas do que aquelas referentes aos dados de consumo alimentar.

O percentual de utilização do SISVAN-Web refere-se ao número de municípios que alimentaram o sistema no período estudado. Considerou-se utilizador do sistema aquele município que realizou acompanhamento nutricional de um ou mais indivíduo(s) no período estudado. O cálculo dessa variável foi obtido por meio da divisão do número de municípios que fizeram uso do aplicativo SISVAN-Web no ano de 2010 pelo número total de municípios que compõem cada CRS. O resultado desse cálculo foi multiplicado por 100.

Cobertura total foi definida como o percentual de indivíduos, de todas as fases do ciclo da vida, acompanhados pelo SISVAN-Web nas Unidades de Saúde em 2010. O cálculo da cobertura total foi obtido por meio da divisão do número de registros do EN nas CRS em 2010 pela população geral das respectivas CRS conforme Censo de $2010^{19}$. O resultado desse cálculo foi multiplicado por 100 para indicar o percentual da população coberta pelo sistema. O mesmo cálculo foi realizado para se obter a cobertura do SISVAN-Web por faixa etária: crianças de 0 a 5 anos, crianças de 5 a 10 anos, adolescentes, adultos e idosos.

Foram coletadas informações demográficas, socioeconômicas e da saúde das $\mathrm{CRS}^{17-20}$. Os dados referentes à área total, população, densidade demográfica e taxa de analfabetismo da população de 15 anos ou mais são provenientes do Censo Demográfico 2010. O PIB per capita de cada CRS foi obtido nas estatísticas da Fundação de Economia e Estatística (FEE ${ }^{20}$. A Taxa de Mortalidade Infantil (TMI) de cada CRS foi calculada por meio da divisão do número de óbitos infantis pelo número de nascidos vivos por local de residência da mãe, sendo o resultado multiplicado por 1000. Os dados utilizados nesse cálculo, assim como a variável cobertura de imunizações, foram obtidos no site do DATASUS ${ }^{18}$. O cálculo da cobertura populacional da Estratégia de Saúde da Família (ESF) foi realizado pela divisão da estimativa da população coberta pela ESF em cada CRS, segundo dados do $\mathrm{DAB}^{17}$, pela população total da respectiva CRS, conforme Censo $2010^{19}$.
O banco de dados foi construído no software Excel 2007 e, posteriormente, transferido e analisado no programa SPSS, versão 17.0. Para a descrição dos dados utilizou-se a média para as variáveis contínuas (densidade demográfica, PIB per capita e taxa de analfabetismo) e frequência para as categóricas (percentual de utilização, cobertura total e por faixa etária). Devido à anormalidade e heterogeneidade dos dados, utilizou-se a análise não paramétrica de Correlação de Spearmann $(r s)$ para verificar a correlação entre as variáveis e os respectivos níveis de significância. Considerou-se estatisticamente significativo $\mathrm{p}$ valor $\leq 0,05$. Os mapas com as coberturas do SISVAN-Web foram elaborados pelo programa TAB para Windows (TabWin), desenvolvido pelo DATASUS. Embora o presente estudo envolva dados relativos a seres humanos, todas as informações utilizadas foram provenientes de base de dados de domínio público e disponíveis nos sites das instituições.

\section{Resultados}

A caracterização demográfica, socioeconômica e de saúde das CRS está descrita na Tabela 1. Observou-se marcada heterogeneidade entre as coordenadorias de saúde no que diz respeito à taxa de analfabetismo (3,3 a 10,0\%), taxa de mortalidade infantil (8,2 a 16,9 por mil) e cobertura da $\operatorname{ESF}(13,1$ a $80,3 \%)$.

Com relação à utilização do SISVAN-Web verificou-se que 324 municípios do RS alimentaram o aplicativo, correspondendo a um percentual de utilização de $65,3 \%$. Conforme ilustrado na Figura 1A, quatro das 19 CRS apresentaram percentuais de utilização acima de $80 \%$, das quais uma teve $100 \%$ de utilização do sistema. A maior parte das coordenadorias $(\mathrm{n}=10)$ apresentou percentuais de utilização entre 60 e $80 \%$, duas mostraram percentuais entre 40 e $60 \%$ e três não chegaram a $40 \%$. No que diz respeito à cobertura total do SISVAN-Web, a quase totalidade $(\mathrm{n}=$ 17) das coordenadorias apresentou coberturas de até 2,5\% (Figura 1B). Os maiores valores de cobertura foram de 6,0\% (CRS 13 - Santa Cruz do Sul) e 3,8\% (CRS 18 - Osório).

A Tabela 2 revela que a cobertura total do SISVAN-Web no RS, em 2010, foi de $1,8 \%$. No que diz respeito à cobertura do SISVAN-Web estratificada por faixa etária, observou-se em todas as CRS, exceto para Santa Maria, uma maior cobertura do sistema nas idades de 0 a 5 anos e de 5 a 10 anos. 
Tabela 1. Caracterização das CRS segundo variáveis demográficas, socioeconômicas e de saúde, no ano de 2010, Rio Grande do Sul, Brasil.

\begin{tabular}{|c|c|c|c|c|c|c|c|c|}
\hline CRS - Sede & $\begin{array}{c}\text { Municípios } \\
\left(\mathbf{n}^{\circ}\right)\end{array}$ & $\begin{array}{l}\text { Área } \\
\text { Total } \\
\left(\mathbf{K m}^{2}\right)\end{array}$ & $\begin{array}{c}\text { Dens. } \\
\text { Demog. } \\
\left(\mathrm{Hab} . / \mathrm{Km}^{2}\right)\end{array}$ & $\begin{array}{c}\text { PIB } \\
\text { per capita } \\
(\mathrm{R} \$)\end{array}$ & $\begin{array}{l}\text { TA } \\
(\%)\end{array}$ & $\begin{array}{c}\text { TMI } \\
\text { (por mil) }\end{array}$ & $\begin{array}{c}\text { Cobertura de } \\
\text { Imunização } \\
(\%)\end{array}$ & $\begin{array}{r}\text { Cobertura } \\
\text { da ESF (\%) }\end{array}$ \\
\hline 1 - Porto Alegre & 24 & $4.604,3$ & 996,7 & $18.820,7$ & 3,3 & 10,8 & 69,4 & 20,8 \\
\hline 2 - Porto Alegre & 41 & $19.275,3$ & 70,8 & $19.172,3$ & 5,8 & 9,0 & 77,1 & 33,2 \\
\hline 3 - Pelotas & 22 & $35.015,7$ & 25,3 & $12.910,5$ & 8,4 & 13,6 & 71,0 & 33,3 \\
\hline 4 - Santa Maria & 31 & $25.413,2$ & 19,7 & $14.663,2$ & 7,0 & 12,7 & 76,8 & 35,8 \\
\hline 5 - Caxias do Sul & 48 & $18.869,2$ & 60,6 & $20.849,7$ & 4,2 & 12,1 & 76,3 & 34,6 \\
\hline 6 - Passo Fundo & 58 & $17.867,2$ & 26,3 & $18.573,3$ & 6,9 & 10,3 & 77,7 & 57,2 \\
\hline 7 - Bagé & 06 & $15.194,4$ & 9,8 & $19.049,3$ & 6,8 & 8,8 & 78,1 & 51,6 \\
\hline 8 - Cachoeira do Sul & 12 & $12.065,5$ & 28,7 & $13.090,0$ & 9,3 & 11,0 & 75,4 & 48,2 \\
\hline 9 - Cruz Alta & 13 & $8.804,2$ & 16,4 & $24.332,1$ & 5,7 & 9,0 & 84,9 & 41,6 \\
\hline 10 - Alegrete & 11 & $41.500,3$ & 8,9 & $18.532,5$ & 7,3 & 16,9 & 74,3 & 13,1 \\
\hline 11 - Erechim & 31 & $5.923,8$ & 28,2 & $15.128,1$ & 6,6 & 12,3 & 77,9 & 67,8 \\
\hline 12 - Santo Ângelo & 25 & $16.512,5$ & 21,8 & $17.218,5$ & 7,6 & 12,5 & 82,8 & 61,1 \\
\hline 13 - Santa Cruz do Sul & 13 & $7.466,7$ & 46,1 & $16.783,2$ & 7,3 & 8,4 & 73,4 & 32,9 \\
\hline 14 - Santa Rosa & 22 & $5.768,8$ & 35,4 & $15.236,3$ & 5,4 & 11,9 & 78,6 & 80,3 \\
\hline 15 - Palmeira das Missões & 26 & $6.341,8$ & 27,4 & $13.662,5$ & 10,0 & 8,9 & 77,8 & 73,4 \\
\hline 16 - Lajeado & 42 & $6.702,1$ & 63,5 & $15.223,8$ & 6,4 & 10,6 & 77,2 & 43,5 \\
\hline 17 - Ijuí & 20 & 7264,5 & 28,0 & $17.103,7$ & 6,5 & 10,0 & 75,7 & 73,0 \\
\hline 18 - Osório & 23 & $8.769,9$ & 95,2 & $11.562,1$ & 7,4 & 10,5 & 81,0 & 46,1 \\
\hline 19 - Frederico Westphalen & 28 & $5.422,1$ & 39,4 & $11.243,9$ & 9,9 & 8,2 & 79,6 & 78,8 \\
\hline Rio Grande do Sul & 496 & $268.781,5$ & 86,7 & $16.481,87$ & 6,9 & 11,2 & 74,1 & 36,0 \\
\hline
\end{tabular}

CRS: Coordenadoria Regional de Saúde; TA: Taxa de Analfabetismo; TMI: Taxa de Mortalidade Infantil.

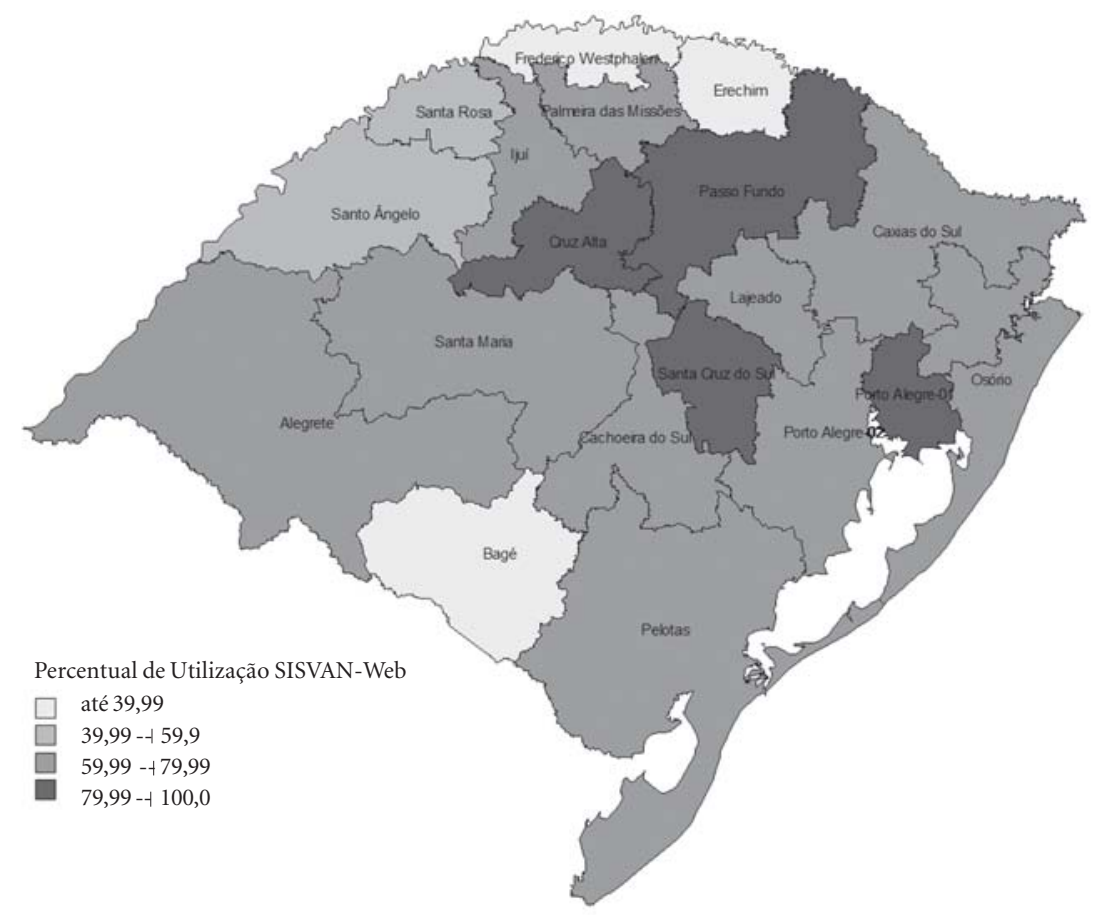

Figura 1A. Percentual de utilização do Sistema de Vigilância Alimentar e Nutricional (SISVAN-Web) por Coordenadoria Regional de Saúde (CRS), no ano de 2010, Rio Grande do Sul, Brasil. 


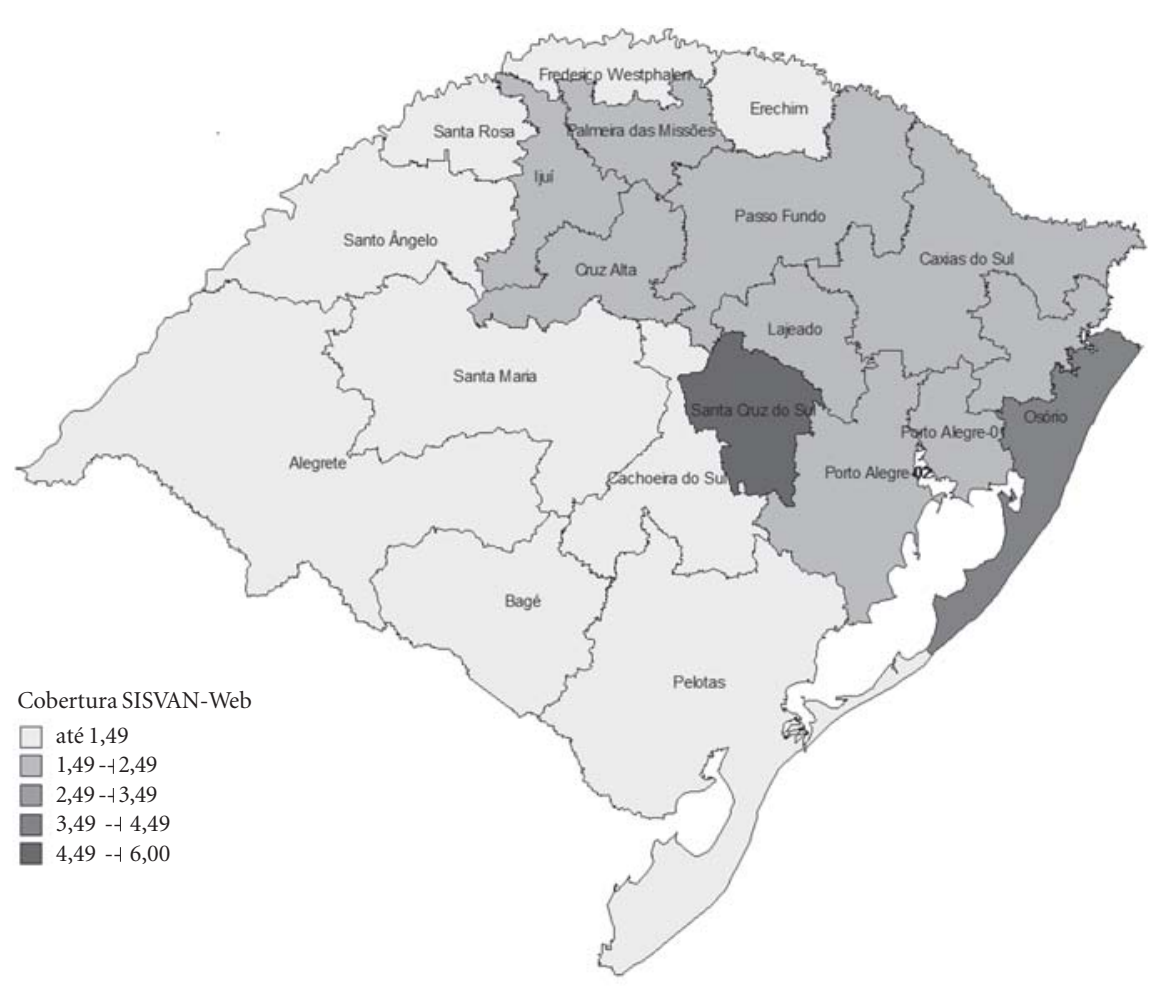

Figura 1B. Cobertura total do Sistema de Vigilância Alimentar e Nutricional (SISVAN-Web) por Coordenadoria Regional de Saúde (CRS), no ano de 2010, Rio Grande do Sul, Brasil.

Tabela 2. Cobertura total e por faixa etária do SISVAN-Web por CRS, no ano de 2010, Rio Grande do Sul, Brasil.

\begin{tabular}{|c|c|c|c|c|c|c|c|}
\hline \multirow[b]{2}{*}{ CRS - Sede } & \multirow[b]{2}{*}{$\begin{array}{c}\text { Municípios } \\
\left(\mathbf{n}^{\mathbf{o}}\right)\end{array}$} & \multicolumn{6}{|c|}{ Cobertura SISVAN-Web } \\
\hline & & $\begin{array}{c}\text { Crianças } \\
0 \text { a } 5 \text { anos } \\
(\%)\end{array}$ & $\begin{array}{c}\text { Crianças } \\
\begin{array}{c}5 \text { a } 10 \text { anos } \\
(\%)\end{array}\end{array}$ & $\begin{array}{c}\text { Adolescentes } \\
(\%)\end{array}$ & $\begin{array}{l}\text { Adultos } \\
\qquad(\%)\end{array}$ & $\begin{array}{c}\text { Idosos } \\
(\%)\end{array}$ & $\begin{array}{c}\text { Total } \\
(\%)\end{array}$ \\
\hline 1 - Porto Alegre & 24 & 9,5 & 6,2 & 1,7 & 0,4 & 0,2 & 1,7 \\
\hline 2 - Porto Alegre & 41 & 4,5 & 4,1 & 1,6 & 1,2 & 0,9 & 1,8 \\
\hline 3 - Pelotas & 22 & 5,2 & 3,2 & 1,2 & 1,0 & 0,1 & 1,3 \\
\hline 4 - Santa Maria & 31 & 2,5 & 4,0 & 1,2 & 0,9 & 1,4 & 1,4 \\
\hline 5 - Caxias do Sul & 48 & 10,7 & 2,5 & 1,3 & 1,2 & 1,6 & 2,2 \\
\hline 6 - Passo Fundo & 58 & 11,6 & 7,0 & 1,7 & 1,0 & 0,8 & 2,2 \\
\hline 7 - Bagé & 06 & 1,7 & 1,1 & 0,1 & 0,1 & 0,0 & 0,3 \\
\hline 8 - Cachoeira do Sul & 12 & 4,3 & 2,4 & 0,6 & 0,5 & 0,3 & 0,9 \\
\hline 9 - Cruz Alta & 13 & 17,4 & 5,1 & 1,6 & 0,6 & 1,3 & 2,3 \\
\hline 10 - Alegrete & 11 & 6,6 & 3,4 & 0,9 & 0,6 & 0,2 & 1,2 \\
\hline 11 - Erechim & 31 & 3,7 & 3,5 & 1,0 & 0,2 & 0,0 & 0,7 \\
\hline 12 - Santo Ângelo & 25 & 1,8 & 1,1 & 0,6 & 0,7 & 0,8 & 0,8 \\
\hline 13 - Santa Cruz do Sul & 13 & 26,6 & 24,6 & 5,3 & 1,7 & 5,4 & 6,0 \\
\hline 14 - Santa Rosa & 22 & 6,5 & 1,5 & 0,6 & 0,2 & 0,6 & 0,8 \\
\hline 15 - Palmeira das Missões & 26 & 8,1 & 7,1 & 1,3 & 0,9 & 0,8 & 1,9 \\
\hline 16 - Lajeado & 42 & 6,5 & 3,8 & 2,2 & 1,7 & 2,4 & 2,3 \\
\hline 17 - Ijuí & 20 & 9,8 & 3,7 & 0,8 & 0,9 & 0,4 & 1,7 \\
\hline 18 - Osório & 23 & 11,7 & 4,6 & 3,1 & 3,0 & 3,5 & 3,8 \\
\hline 19 - Frederico Westphalen & 28 & 5,8 & 3,9 & 0,7 & 0,6 & 0,2 & 1,2 \\
\hline Rio Grande do Sul & 496 & 10,5 & 5,1 & 1,6 & 0,8 & 0,9 & 1,8 \\
\hline
\end{tabular}


Verificaram-se, ainda, importantes diferenças entre as CRS no que se refere à cobertura de crianças de 0 a 5 anos, variando de $1,7 \%$ (CRS 07 - Bagé) a 26,6\% (CRS 13 - Santa Cruz do Sul). Entretanto, a maioria $(\mathrm{n}=14)$ das coordenarias apresentou valores de cobertura para essa faixa etária abaixo de $10 \%$. Quanto à cobertura SISVAN-Web entre crianças de 5 a 10 anos, constatou-se que todas as CRS apresentaram coberturas menores que $8 \%$, com exceção de uma, que obteve uma cobertura de aproximadamente 25\% (CRS 13 - Santa Cruz do Sul).

Ainda na Tabela 2 verifica-se que o grupo de adolescentes apresentou baixos valores de cobertura. Quase a totalidade das coordenadorias ( $\mathrm{n}$ $=18$ ) apresentou valores de até $2,5 \%$; oito dessas obtiveram coberturas que não chegaram a $1 \%$. Comportamento semelhante foi observado no grupo de idosos. Para essa faixa etária, a cobertura média foi de $0,9 \%$, variando de zero a $5,4 \%$. Treze das 19 CRS não alcançaram cobertura de $1 \%$. Com relação à população adulta, a cobertura do sistema encontrou-se abaixo de $3 \%$ em todas as CRS. É importante ressaltar que $83,2 \%$ dos adultos avaliados eram do sexo feminino.

A Tabela 3 mostra a correlação entre percentual de utilização e cobertura do SISVAN-Web e as variáveis demográficas, socioeconômicas e de saúde das CRS. Verificou-se relação significativa entre o percentual de utilização do SISVAN-Web e a cobertura da ESF em cada CRS, isto é, à medida que aumentavam os percentuais de cobertura da ESF, diminuía a utilização do aplicativo $(r s=-0,685 ; \mathrm{p}=0,01)$. As correlações entre o percentual de utilização e as demais variáveis não apresentaram diferença estatística. As correlações entre a cobertura total do SISVAN-Web e as variáveis demográficas, socioeconômicas e de saúde das CRS não foram significativas.

\section{Discussão}

Considerando-se a escassez de pesquisas avaliativas acerca do desempenho do SISVAN, pode-se dizer que o presente estudo viabilizou uma descrição ainda pouco explorada sobre a utilização e a cobertura do aplicativo SISVAN-Web em um estado brasileiro. Entretanto, algumas limitações precisam ser consideradas. A primeira está relacionada aos potenciais vieses inerentes ao uso de dados secundários, tais como diversidade na confiabilidade da informação, sub-registro e erros decorrentes da coleta, preenchimento e digitação dos dados. A segunda limitação decorre do viés metodológico do delineamento do estudo por si só, que pode levar a falácias ecológicas, como talvez a associação entre menor utilização do SISVAN-Web em CRS com maior cobertura de ESF.

Os resultados indicam baixos percentuais de utilização $(65,3 \%)$ do SISVAN-Web no estado do RS em 2010. Similarmente, em 2006, Damé et al. ${ }^{21}$ encontrou, no RS, uma taxa de utilização do SISVAN-MS, aplicativo do DATASUS vigente na época, de $61,3 \%$. Os dados consolidados mostram que apenas uma das 19 coordenadorias utilizou

Tabela 3. Correlação de Spearman entre percentual de utilização e cobertura total SISVAN-Web em relação às variáveis geográficas, socioeconômicas e de saúde, no ano de 2010, Rio Grande do Sul, Brasil.

\begin{tabular}{lcc}
\hline \multicolumn{1}{c}{ Variáveis geográficas, socioeconômicas e de saúde } & Correlação de Spearmann & p \\
\hline Percentual de utilização SISVAN-Web & & 0,347 \\
Área Total $\left(\mathrm{Km}^{2}\right)$ & 0,228 & 0,472 \\
Dens. Demog. (Hab./Km²) & 0,176 & 0,151 \\
PIB Percapita (R\$) & 0,342 & 0,414 \\
Taxa de Analfabetismo & $-0,199$ & 0,652 \\
Taxa de Mortalidade Infantil (TMI) & $-0,111$ & 0,128 \\
Cobertura de Imunização & $-0,362$ & 0,001 \\
Cobertura Estratégia de Saúde da Família & $-0,685$ & 0,989 \\
Cobertura total SISVAN-Web & & 0,071 \\
Área Total (Km ${ }^{2}$ ) & 0,004 & 0,622 \\
Dens. Demog. (Hab./Km $\left.{ }^{2}\right)$ & 0,423 & 0,552 \\
PIB per capita (R\$) & 0,121 & 0,135 \\
Taxa de Analfabetismo & $-0,146$ & 0,596 \\
Taxa de Mortalidade Infantil (TMI) & $-0,356$ & 0,104 \\
Cobertura de Imunização & $-0,130$ & $-0,384$ \\
Cobertura Estratégia de Saúde da Família & & \\
\hline
\end{tabular}


o aplicativo em $100 \%$ dos seus municípios. Importante destacar, no entanto, que foram considerados utilizadores do sistema aqueles municípios que realizaram acompanhamento nutricional de pelo menos um indivíduo. Dessa forma, sugere-se cautela ao observar esse resultado isoladamente, uma vez que embora tenha obtido o melhor desempenho no indicador utilização, a CRS apresentou uma taxa de cobertura pouco expressiva, a exemplo das demais.

Frente às dificuldades de consolidação do SISVAN, a Coordenação-Geral da Política Nacional de Alimentação e Nutrição (CGPAN) desencadeou, em 2006, um processo de reavaliação e replanejamento das ações de VAN. As oficinas de trabalho realizadas resultaram no reconhecimento das limitações e dificuldades de execução das ações de vigilância em sua plenitude. As perspectivas de mudança apontadas na ocasião culminaram no lançamento, em dezembro de 2007, do aplicativo SISVAN-Web ${ }^{6}$. Levando-se em consideração os esforços envidados para a ampliação e a qualificação da VAN, pondera-se que as diferenças nas taxas de utilização no RS de 2006 para 2010 são inexpressivas quando se almeja uma maior identificação das situações de risco nutricional no âmbito do SUS. Cabe destacar que os dados de consumo alimentar no SISVAN-Web não foram analisados no presente estudo em razão da incipiência de seus registros no sistema. Sendo assim, supõe-se que a ampliação da análise nesse sentido evidenciaria ainda mais a subutilização do sistema.

Considerando-se a complexidade de implantação do SISVAN, é possível supor que a dificuldade de adesão ao sistema não seja exclusividade do RS. Outros dois estudos realizados em São Paulo encontraram resultados semelhantes. As taxas de utilização do SISVAN/SP nos anos de 2001 e 2003 foram de $58 \%$ e $74,7 \%$, respectivamente $^{22,23}$. Segundo os autores, em decorrência da implantação do aplicativo ter sido iniciada em 2001, três das vinte e quatro Diretorias Regionais de Saúde (DIR) do estado de São Paulo apresentaram taxa de utilização de $0 \%$ naquele $a^{22}$. Embora sejam inquestionáveis os baixos percentuais de utilização do SISVAN-Web no RS em 2010, Romeiro ${ }^{3}$ apontou a região Sul como aquela com melhor grau de implantação (46,5\%) do sistema em todo o País no ano de 2006. Esse estudo definiu o grau de implantação do SISVAN por meio de um modelo validado de classificação (crítico, aceitável e satisfatório). Conforme os autores, nenhuma região brasileira apresentou grau de implantação satisfatório então.
Logo, percebe-se que as dificuldades de consolidação do SISVAN extrapolam as diferenças regionais, reforçando a concepção de que a efetivação das ações de VAN demanda uma profunda reflexão acerca dos aspectos técnico-operacionais desse sistema.

A análise da cobertura total do SISVAN-Web, em 2010, revela valores pouco expressivos. Desde 1993, a baixa cobertura geográfica e populacional do SISVAN, assim como a subutilização dos dados, vinha sendo apontada como um problema permanente que compromete o conceito de VAN $^{24}$. Sabe-se que um sistema de VAN sustenta-se quando fornece dados úteis à tomada de decisão de modo a subsidiar a formulação das políticas públicas e o desenho dos programas de nutrição. Por dados úteis entende-se uma informação confiável e representativa ${ }^{24,25}$. Nesse contexto, a cobertura do sistema destaca-se como um dos mecanismos de avaliação e aprimoramento da representatividade dos dados gerados ${ }^{25}$. De fato, com uma cobertura total de apenas $1,84 \%$, a representatividade dos dados provenientes do SISVAN-Web torna-se questionável. Portanto, é recomendável cautela na avaliação do estado nutricional e na extrapolação desses resultados para a população em geral ${ }^{13}$.

Diversos estudos ${ }^{13,14,23,25}$ levantam hipóteses que podem, de certa forma, justificar os baixos percentuais de utilização e cobertura do SISVANWeb encontrados no Rio Grande do Sul em 2010: descompasso entre o discurso político e a priorização da alimentação e nutrição nas políticas governamentais; sistema demasiadamente complexo que o torna inoperável frente às condições e disponibilidades institucionais; criação de uma estrutura técnico-administrativa exclusiva, o que o torna um núcleo isolado frente aos demais setores da instituição onde se insere; subestimação da estrutura física e de recursos humanos necessários à operacionalização do sistema localmente; dificuldades dos profissionais em relação à tomada de medidas antropométricas; falta de comunicação com o sistema de vigilância epidemiológica; divulgação e discussão insuficiente dos dados que gera nas três esferas do sistema de saúde; competição das ações de VAN com as demais atividades da atenção básica em nível local; dificuldade em registrar sistematicamente as informações em planilhas, assim como em digitar os dados no sistema; dentre outros.

Sobre o acompanhamento nutricional do público infantil, constatou-se que a cobertura do SISVAN-Web para a população de crianças menores de cinco anos foi de aproximadamente 
10,5\%. Cobertura semelhante foi observada em $\mathrm{SP}^{22}$, no ano de 2001, quando em média, 14,5\% desse público foi avaliado pelo SISVAN/SP. Os autores chamam a atenção para o fato de que esse valor corresponde à cobertura das quatro Diretorias Regionais (DIR) com maiores percentuais de utilização, ou seja, aquelas com mais de 90\% de municípios com o aplicativo implantado. Dessa forma, se a análise da cobertura fosse realizada com base em todo o estado, ou seja, considerando-se todas as DIR, inclusive aquelas com $0 \%$ de percentual de utilização, a cobertura real do sistema para essa faixa etária mostrarse-ia menor.

Constatou-se, no presente estudo, que a cobertura do SISVAN-Web entre crianças menores de 10 anos foi menor do que aquela encontrada (10,5\%) em 2006 por Damé et al. ${ }^{21}$. Entretanto, para o cálculo da cobertura os autores utilizaram como denominador a população de 0 a 10 anos coberta pela Estratégia de Saúde da Família (ESF). Cabe ressaltar que a população monitorada por meio das ações de VAN é formada tanto por indivíduos que procuram por demanda espontânea os Estabelecimentos Assistenciais de Saúde (EAS) quanto por aqueles assistidos pelos programas de Saúde da Família (PSF) e Agentes Comunitários de Saúde (PACS) ${ }^{3}$. Considerando-se a baixa cobertura de Saúde da Família no RS, uma das hipóteses para a superestimação da cobertura do sistema em $2006^{21}$ é o cálculo inexato da população beneficiária do SISVAN.

Em decorrência do rápido processo de transição alimentar e nutricional que o Brasil vivenciou e vivencia, a evolução gradual dos problemas de saúde processa alterações no modo de adoecer e morrer da população, universalizando-se uma pauta variada de demandas para todas as idades. Dessa forma, diferentemente dos demais países das Américas, no Brasil, o sistema amplia a abordagem de VAN uma vez que está preparado para receber dados de monitoramento de todas as fases do curso da vida, extrapolando o público materno-infantil como era no início $^{3}$. Entretanto, os dados nacionais do SISVAN apontam que grande parte dos registros é realizada com crianças. Segundo o Ministério da Saúde, o público infantil representa mais da metade dos registros do sistema nos anos de 2003 a 2006. (96,4\%, 90,04, 69,3\% e 58,4\%, respectivamente $)^{13}$. A observação de que o público infantil é responsável pela preponderância de registros fez-se verdadeira nos achados deste estudo. Levando-se em consideração que a recomendação nacional é que as ações sejam iniciadas pelo pú- blico materno-infantil, é possível concluir que o SISVAN, apesar dos avanços descritos na literatura $^{6}$, ainda encontra-se em estágio inicial de sua implantação.

Acerca da população adolescente, constatouse uma cobertura do SISVAN-Web de apenas $1,6 \%$. Assim como nos adultos, entre os adolescentes, a obesidade, a hipertensão arterial e as dislipidemias já revelam-se como problemas importantes de saúde e se mostram fortemente associadas às condições de nutrição e ao estilo de vida adotado e/ou imposto pela sociedade moderna $^{26}$. Dessa forma, torna-se necessário que os profissionais e as equipes de saúde, assim como os gestores do SUS nas diferentes esferas, encontrem meios de efetivar o acompanhamento nutricional sistemático dessa população.

A análise da cobertura do SISVAN-Web para adultos mostrou que apenas $0,82 \%$ dessa população teve seu estado nutricional registrado, sendo que a maior parte refere-se à população feminina. Em todas as regiões do país vem crescendo o contingente de adultos que apresentam sobrepeso e obesidade. Entre a população adulta, destaca-se a elevação das taxas de doenças como diabetes, obesidade, neoplasia, hipertensão arterial e dislipidemias. Em termos relativos, a situação mais crítica é verificada na região Sul, especialmente para os homens uma vez que para esses a prevalência de excesso de peso é maior ${ }^{26,27}$. Diante disso, evidencia-se a necessidade de avaliar as estratégias utilizadas pelos serviços de saúde para captar a população adulta, principalmente a masculina.

Chama a atenção, no presente estudo, que a utilização do SISVAN-Web é reduzida na medida em que aumenta a cobertura da ESF na CRS, achado contrário ao esperado. Apesar da ESF ser considerada um modelo facilitador do cumprimento da agenda da APS, no que diz respeito à execução das atividades de VAN, a premissa não demonstrou-se verdadeira neste estudo. A Política Nacional de Atenção Básica (PNAB) tem na Saúde da Família sua estratégia prioritária para expansão e consolidação da APS. Para tanto, a ESF atua a partir da organização de equipes de saúde da família, integradas idealmente por um médico, um enfermeiro, um auxiliar de enfermagem e Agentes Comunitários de Saúde (ACS), embora se pressuponha que outros profissionais possam ser incorporados de acordo com a demanda dos serviços. A estas equipes são colocados os desafios de compreender melhor o processo saúde/doença experimentado pela população de sua área de atuação e também a tare- 
fa de ir além da prática curativa tradicional ${ }^{26}$. Nesse sentido, as equipes de Saú-de da Família deveriam ser incentivadas a realizar ações de nutrição em seus territórios, incluindo o monitoramento da situação alimentar e nutricional da população atendida.

\section{Considerações finais}

Os resultados deste estudo indicaram baixos percentuais de utilização e cobertura do SISVANWeb no Rio Grande do Sul em 2010, evidenciando que a cultura de monitorar o estado nutricional da população resiste em inserir-se na pauta de ações das equipes de saúde da APS. A afirmativa de que a VAN é mais consentida do que estimulada foi ratificada no presente estudo. Conforme Batista-Filho e Rissin ${ }^{24}$, os indicadores de saúde tradicionais possuem um espaço culturalmente bem definido, de modo que a VAN tornase um trabalho de diletantismo técnico, ainda sem a necessária acústica política junto às autoridades e ao público.

Valorizar o estado nutricional, assim como o registro adequado dos dados, é ratificar a importância da nutrição como coadjuvante das ações básicas de saúde, consolidando sua vocação como sistema de informação de base local ${ }^{28,29}$.
A ausência de um monitoramento do estado nutricional da população brasileira não condiz com o estágio de desenvolvimento econômico alcançado pelo País, que necessitaria de um sistema de informação ágil e de qualidade para estar sistematicamente revendo suas diretrizes na política de saúde e nutrição ${ }^{2}$.

Este trabalho poderá subsidiar a realização de estudos similares em outros estados brasileiros, de modo que as informações geradas possam servir como base para aprimoramento do SISVAN-Web. Servirá, outrossim, como instrumento norteador do planejamento de ações estratégicas de VAN na APS, como tentativa de reverter a baixa utilização e cobertura do sistema e acompanhar, periodicamente, a evolução dessa vigilância. Espera-se, ainda, que os resultados deste estudo possam sensibilizar os profissionais da saúde para a atitude de vigilância nutricional, tão importante para o direcionamento dos programas de saúde vigentes.

Por fim, aponta-se a necessidade de mais estudos sobre o SISVAN-Web no que diz respeito ao seu desempenho como um sistema de vigilância. Recomenda-se como de extrema importância a realização de estudos qualitativos uma vez que essa metodologia permitirá uma maior compreensão dos fatores que dificultam a implementação desse sistema.

\section{Colaboradores}

NM Jung, FS Bairros e MB Neutzling participaram igualmente de todas as etapas de elaboração do artigo. 


\section{Referências}

1. Brasil. Ministério da Saúde (MS). Secretaria de Atenção à Saúde. Departamento de Atenção Básica. Brasil. Política Nacional de Alimentação e Nutrição. Brasília: MS; 2012.

2. Leal MC, Bittencourt SA. Informações nutricionais: o que se tem no país? Cad Saude Publica 1997; 13(3): 551-555

3. Romeiro AAF. Avaliação da Implantação do Sistema de Vigilância Alimentar e Nutricional - SISVAN, no Brasil [dissertação]. Brasília: Universidade de Brasília; 2006.

4. Lamounier JA. Transição epidemiológica nutricional em crianças e adolescentes argentinos de áreas carentes. Revista Paulista de Pediatria 2009; 95(2):124-126.

5. Batista Filho M, Rissin A. A transição nutricional no Brasil: tendências regionais e temporais. Cad Saude Publica 2003; 19(1):181-191.

6. Coutinho JG, Toral N, Ubarana JA, Aquino KKNC, Nilson EAF, Fagundes A, Vasconcellos AB. A organização da Vigilância Alimentar e Nutricional no Sistema Único de Saúde: histórico e desafios atuais. Revista Brasileira de Epidemiologia 2009; 12(4):688-699.

7. Hartz ZMA. Meta-Avaliação da gestão em saúde: desafios para uma "nova saúde pública". Cien Saude Colet 2011; 17(4):832-834.

8. Brasil. Ministério da Saúde (MS). Secretaria de Atenção à Saúde. Departamento de Atenção Básica. Brasil. Avaliação na Atenção Básica em Saúde: caminhos da institucionalização. Brasília: MS; 2005.

9. Santos IS. Avaliação do impacto de programas nutricionais. Revista de Nutrição 2009; 22(1):141-150.

10. Allen LH, Gillespie SR. What Works? A Review of the Efficacy and Effectiveness of Nutrition Interventions. Manila: WHO; 2001.

11. Costa JMBS, Felisberto E, Bezerra LCA, Cesse EAP, Samico IC. Monitoramento do desempenho da gestão da vigilância em saúde: instrumentos e estratégias de uso. Cad Saude Publica 2013; 18(5):1201-1216.

12. Brasil. Ministério da Saúde (MS). CoordenaçãoGeral da Política de Alimentação e Nutrição C. Relatório de Gestão 2007-2010. Brasília: MS; 2011.

13. Brasil. Ministério da Saúde (MS). Secretaria de Atenção à Saúde. Departamento de Atenção Básica. Indicadores de Vigilância Alimentar e Nutricional. Brasília: Ministério da Saúde; 2009.

14. Acosta SJ. Errores en que se puede incurrir en los sistemas de vigilancia alimentaria y nutricional. $R e-$ vista Cubana de Alimentación y Nutrición 2001; 15(1):68-73.

15. Santos NM. A organização da saúde no Rio Grande do Sul. Revista Gestão \& Saúde 2010; 1(3):1-10.

16. Sisvan-Web. Sisvan-Web: Módulo Gerador de Relatórios. [Internet]. [acessado 2012 mar 14]. Disponível em: http://nutricao.saude.gov.br/sisvan/relatorios _publicos/rel_consolidado_acompanhamento.php

17. Departamento de Atenção Básica do Ministério da Saúde. Evolução do credenciamento e implantação do estratégia Saúde da Família. [Internet]. [acessado 2014 mar 28]. Disponível em: http://dab.saude. gov.br/historico_cobertura_sf.php
18. Departamento de Informática do SUS. Informações de Saúde DATASUS. [Internet]. [acessado 2014 abr 14]. Disponível em: http://www2.datasus.gov.br/DATASUS/index.php?area $=02$

19. Instituto Brasileiro de Geografia e Estatística. Censo 2010. [Internet]. [acessado 2012 abr 14]. Disponível em: http://www.censo2010.ibge.gov.br/resultados_ do_censo2010.php

20. Fundação de Economia e Estatística. PIB Municipal - Série Histórica. [Internet]. [acessado 2012 mar 28]. Disponível em: http://www.fee.tche.br/sitefee/pt/ content/estatisticas/pg_pib_municipal_sh.php

21. Damé PKV, Pedroso MRO, Marinho CL, Gonçalves VM, Duncan BB, Fisher PD, Romero ALC, Castro TG. Sistema de Vigilância Alimentar e Nutricional (SISVAN) em crianças do Rio Grande do Sul, Brasil: cobertura, estado nutricional e confiabilidade dos dados. Cad Saude Publica 2011; 27(11):2155-2165.

22. Lei DLM, Chaves SP, Saldiva SRDM, Stefanini MLR. Sistema de Vigilância Alimentar e Nutricional no Estado de São Paulo. Boletim do Instituto de Saúde 2002; 26:1-24

23. Venâncio SI, Levy RB, Saldiva SRDM, Mondini L, Stefanini MLR. Sistema de vigilância alimentar e nutricional no Estado de São Paulo, Brasil: experiência da implementação e avaliação do estado nutricional de crianças. Rev Brasileira Saúde Materno Infantil 2007; 7(2):213-220.

24. Batista-filho M, Rissin A. Vigilância Alimentar e Nutricional: antecedentes, Objetivos e Modalidades. A VAN no Brasil. Cad Saude Publica 1993; 9(1): 99-105.

25. Arruda BKGD. Sistema de Vigilância Alimentar e Nutricional. Frustrações, Desafios e Perspectivas. Cad Saude Publica 1992; 8(1):96-101.

26. Assis AMO, Santos SMC, Freitas MCS, Santos JM, Silva MCM. O Programa Saúde da Família: contribuições para uma reflexão sobre a inserção do nutricionista na equipe multidisciplinar. Rev Nutrição 2002; 15(3):255-266.

27. Tardido AP, Falcão MC. O impacto da modernização na transição nutricional e obesidade. Revista Brasileira de Nutrição Clínica 2006; 21(2):117-124.

28. Brasil. Ministério da Saúde (MS). CoordenaçãoGeral da Política Nacional de Alimentação e Nutrição. Departamento de Atenção Básica. Brasil. Vigilância Alimentar e Nutricional - SISVAN: orientações básicas para a coleta, o processamento, a análise de dados e a informação em serviços de saúde. Brasília: MS; 2004.

29. Recine E, Vasconcellos AB. Políticas nacionais e o campo da Alimentação e Nutrição em Saúde Coletiva: cenário atual. Cad Saude Publica 2011; 16(1):7379.

Artigo apresentado em 10/08/2013

Aprovado em 10/10/2013

Versão final apresentada em 18/10/2013 